\title{
Trends in birth prevalence of cerebral palsy
}

\author{
P O D PHAROAH, T COOKE, I ROSENBLOOM, AND R W I COOKE \\ Departments of Community Health and Child Health, University of Liverpool
}

SUMMARY A register of children with cerebral palsy born in the period 1966-77 to mothers resident in the Mersey region was compiled from several different data sources. There were 685 cases, with a male:female ratio of $1 \cdot 4: 1$. The birth prevalence of cerebral palsy ranged from $1 \cdot 18$ to 1.97 per 1000 live births each year, with a mean of 1.51 per 1000 live births. There was no discernible trend in overall prevalence, but there was a highly significant upward trend in the prevalence of cerebral palsy among low birthweight infants, which was evident for all the major types of cerebral palsy. It is postulated that this is due to improved survival rather than a real change in incidence. Alternatively, because the register was compiled from health service records, a diagnostic access bias from improved follow up of low birthweight infants may have led to an apparent increase in prevalence.

Cerebral palsy has been defined as comprising 'a group of disorders of movement and posture due to a defect or lesion of the immature brain'. ${ }^{1}$ In recent years there has been mounting interest in cerebral palsy, particularly in possibilities for its prevention. In part this interest has been precipitated by the improvement in survival in low birthweight infants, which has paralleled the development of special and intensive care nurseries. ${ }^{2}{ }^{3}$ In Little's classic description over a century ago the common association of premature birth with cerebral palsy was recognised. ${ }^{4}$ While modern neonatal intensive care has been in the forefront of medical advance and is associated with improved survival, there is still speculation that the improvement may only be at the cost of an increase in prevalence of cerebral palsy and other disabilities. ${ }^{5-7}$ It is for this reason that the monitoring of cerebral palsy epidemiology in conjunction with mortality statistics has been suggested as an indicator of the gains and hazards of perinatal care. $^{8}$

Recent studies examining birthweight specific trends in the prevalence of cerebral palsy have shown conflicting trends. In Sweden there was a fall in the overall trend between 1954 and 1970 from $2 \cdot 2$ to 1.3 per 1000 live births, due mainly to a decrease in the syndromes of spastic and ataxic diplegia, particularly among low birthweight babies and in the group with perinatal causes of cerebral palsy. ${ }^{9}$ Subsequently, however, there was an increase in prevalence to 2.0 per 1000 in the period $1975-78$, which was mainly due to spastic/ataxic diplegias among preterm infants. ${ }^{10}$ The series from Western Australia covered the period 1956-75; the observations differed in two important respects from the Swedish study. There was a significant rise in the prevalence in the 1966-70 period followed by a highly significant fall in the 1971-75 period, which was of equal magnitude in infants of low $(\leqslant 2500 \mathrm{~g})$ and normal $(>2500 \mathrm{~g})$ weight at birth. ${ }^{11}$ The most recent study from the north east of England, covering the period $1960-75$, found a decrease in prevalence among very low birthweight babies but an increase among babies weighing more than $2500 \mathrm{~g}$ at birth. ${ }^{12}$

We report here an examination of the trends in the birthweight specific prevalence of cerebral palsy at birth in the Mersey region from 1966 to 1977.

\section{Patients and methods}

A register was compiled of all cases of cerebral palsy born in the years 1966-77 whose mother's area of residence at the time of birth was within the boundary of the Mersey Regional Health Authority. The 1974 National Health Service and Local Authority reorganisation was associated with the redrawing of geographical boundaries. Before 1974, those county councils and boroughs and urban and rural districts that subsequently constituted the Mersey region after the 1974 reorganisation provided the geographical delineation of the study area. As the mother's area of residence at the time of birth was the criterion for inclusion infants born 
within the region but whose mother's area of residence was outside were excluded; those born outside but whose mother's area of residence was within the region were included. These inclusion and exclusion criteria were applied because denominator data for live births obtained from birth registration procedures are published according to the mother's area of residence at the time of birth.

To ensure completeness of ascertainment of cases, several sources of information were used. Handicap registers in each of the 10 health districts in the region were abstracted for all cases with a diagnosis of cerebral palsy or possible cerebral palsy. In addition, a list of all cases of the disease resident in the Mersey region was obtained from the national register of families claiming a family disability allowance, which is maintained by the Rowntree Trust at York. Records of all children attending schools for the educationally subnormal (moderate and severe) and the physically handicapped were inspected and any child with a diagnosis of cerebral palsy was noted. Lastly, through the Office of Population Censuses and Surveys (OPCS), a search was made for all death certificates in which the disease featured as the underlying cause of death on Part I or as other significant condition contributing to the death on Part II of the certificate.

The categorisation of type of cerebral palsy was made on the basis of information in the clinical records of each child. The records of all doubtful cases were reviewed by one of us (LR). A case was classified as 'acquired' if the presumed insult to the brain occurred after the 28 th day of life. If there was uncertainty about the timing of the insult the aetiology was presumed congenital. All acquired cases were excluded from subsequent analysis. Cases with symmetrical or nearly symmetrical spasticity of the legs with lesser involvement of either arm were designated as having spastic diplegia. Cases with unilateral spasticity of one arm and one leg were designated as hemiplegia and those with arms and legs equally affected or with deficits greater in the arms than the legs as spastic quadriplegia. If, in addition to the spasticity, dyskinesia or ataxia was present the child was labelled as a 'mixed' case. The remaining cases designated 'other' included the athetoid, ataxic, and dystonic forms.

Denominator data for determining birth prevalences of cerebral palsy were obtained from the Registrar General's and OPCS tables of birth registration. Grouped birthweight specific data for infants weighing $2500 \mathrm{~g}$ or less were obtained from the LHS $27 / 1$ returns compiled by the Department of Health and Social Security (DHSS) from birth notifications. Numbers of live births of infants weighing over $2500 \mathrm{~g}$ were obtained by subtracting birth notifications $2500 \mathrm{~g}$ or less from total birth registrations.

There were anomalies in using the LHS 27/1 returns to obtain denominators for determining birthweight specific birth prevalences of cerebral palsy. Firstly, these data for 1966 were not available as the regional records had been destroyed. Secondly, it was not possible to match precisely the boundaries covered by the LHS 27/1 returns before and after the 1974 health service reorganisation. After 1974 the Mersey Regional Health Authority included part of what was previously Lancashire County Borough and excluded part of what was previously Cheshire County Borough. These changes have been allowed for by the addition or subtraction of the appropriate county borough health division data on low birthweight infants for the years 1967-73.

\section{Results}

A total of 870 cases of cerebral palsy born in the years 1966-77 inclusive to mothers resident in the Mersey region comprised the register. Of these, 185 that were 'acquired' were excluded. All subsequent analyses relate to the remaining 685 cases, of which 403 were boys and 282 girls, a male:female ratio of $1 \cdot 4: 1$

Type of cerebral palsy. The distribution of the cases according to type of cerebral palsy is shown in Table 1. There were 19 cases unclassified because of death or loss to follow up before definitive clinical details were recorded.

Completeness of ascertainment. A failure of ascertainment may have been due to death or loss to follow up before the diagnosis was made. Under

Table 1 Distribution of the 685 cases (after 185 'acquired' cases had been excluded) by type of cerebral palsy

\begin{tabular}{lrr}
\hline Type of cerebral palsy & \multicolumn{2}{l}{$\begin{array}{l}\text { No }(\%) \\
\text { of cases }\end{array}$} \\
\hline Spastic: & 185 & $(27 \cdot 0)$ \\
$\quad$ Hemiplegic & 144 & $(21 \cdot 0)$ \\
Diplegic & 190 & $(27 \cdot 7)$ \\
Quadriplegic & 12 & $(1 \cdot 8)$ \\
Mixed Hemiplegic & 5 & $(0 \cdot 7)$ \\
Mixed diplegic & 57 & $(8 \cdot 3)$ \\
Mixed quadriplegic & 28 & $(4 \cdot 1)$ \\
Dyskinetic & 29 & $(4 \cdot 2)$ \\
Ataxic & 2 & $(0 \cdot 3)$ \\
Ataxic/dyskinetic & 14 & $(2 \cdot 0)$ \\
Hypotonic & 19 & $(2 \cdot 8)$ \\
Unclassified & 685 & $(100)$ \\
\hline Total & & \\
\hline
\end{tabular}


recording of the latter was usually due to migration. From our records it was possible to identify 71 cases whose mothers migrated into the Mersey region after the birth of the affected child. (These cases were not part of the 685 children who were the basis of the analysis in this paper.) In contrast, there were only 38 cases who migrated out of the region after birth. The total population of the region altered little

Table 2 Annual birthweight specific frequencies and prevalences of cerebral palsy in the Mersey region 1966-77

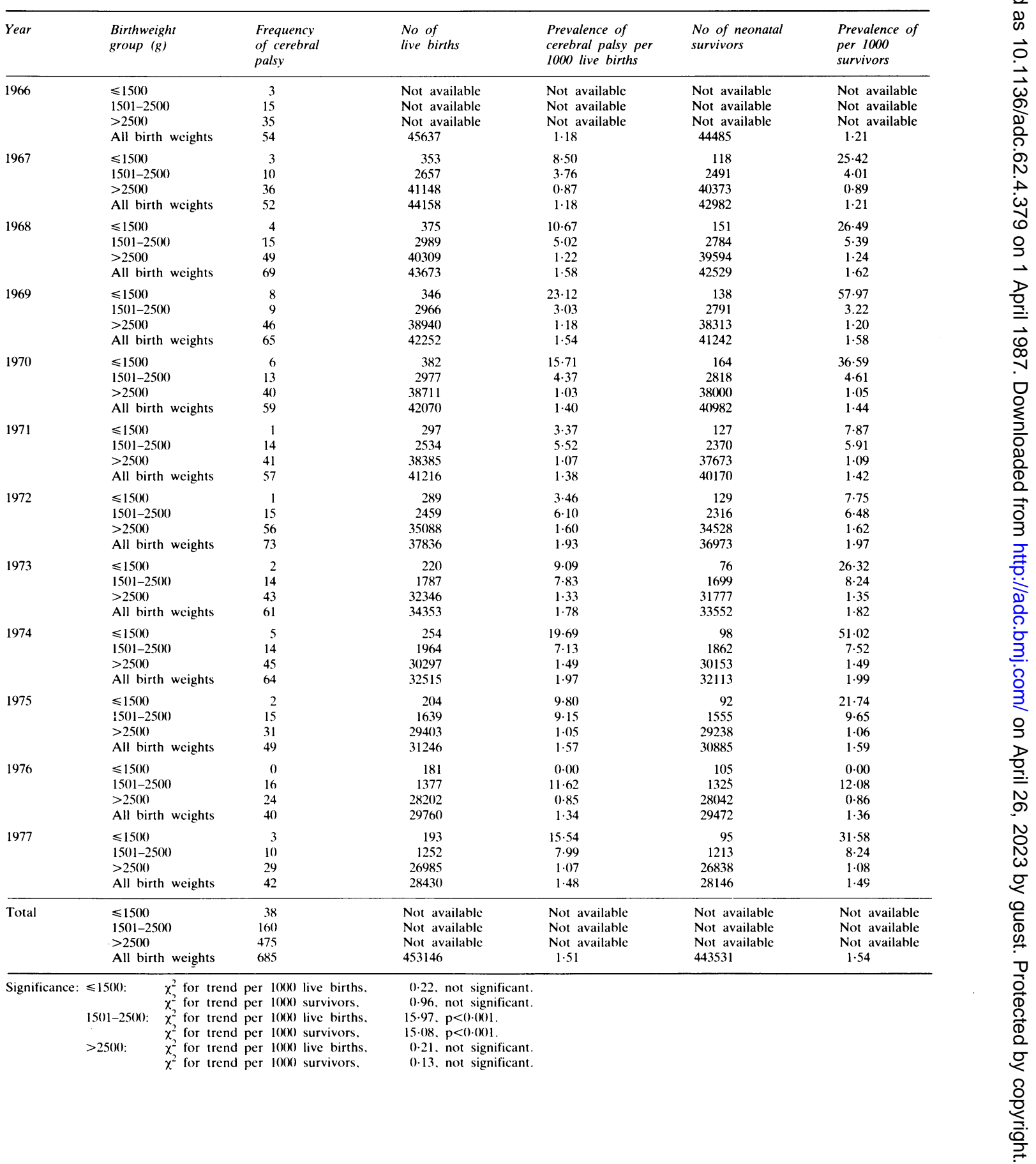


during the study; there was a small increase in population in the early years followed by a compensatory decrease. Therefore, the recorded difference in cases of cerebral palsy between migration in and migration out of the region was presumably an artefact due to incomplete ascertainment of those who migrated out, unless there was a differential migration of cases with cerebral palsy relative to the total population. Examination of this discrepancy by year of birth showed that it was evenly spread over the 12 year sfudy period, which means that it is unlikely to have led to a bias in the trends observed.

Trends in the birth prevalence of cerebral palsy. There were 453146 live births in the Mersey region during the years 1966-77 inclusive, with 685 cases of cerebral palsy, giving an overall birth prevalence of 1.51 per 1000 live births. Annually, the birth prevalence ranged between 1.18 and 1.97 per 1000 live births, but there was no discernible trend over the period studied $\left(\chi_{\mathrm{IDH}}^{2}\right.$ for trend $2 \cdot 21$, not significant).

Table 2 shows annual birthweight specific frequencies and prevalences for cerebral palsy. The 54 cases born in 1966 had to be excluded from the analysis because birthweight specific denominator data for the region were not available, the LHS 27/1 records having been destroyed. An additional 11 cases born in the years 1967-77 inclusive also had to be excluded as their birth weight was not recorded. Trends in birthweight specific prevalences are shown in Figure 1 for two groups, infants with birth weights $\leqslant 2500 \mathrm{~g}$ and $>2500 \mathrm{~g}$. There was significant

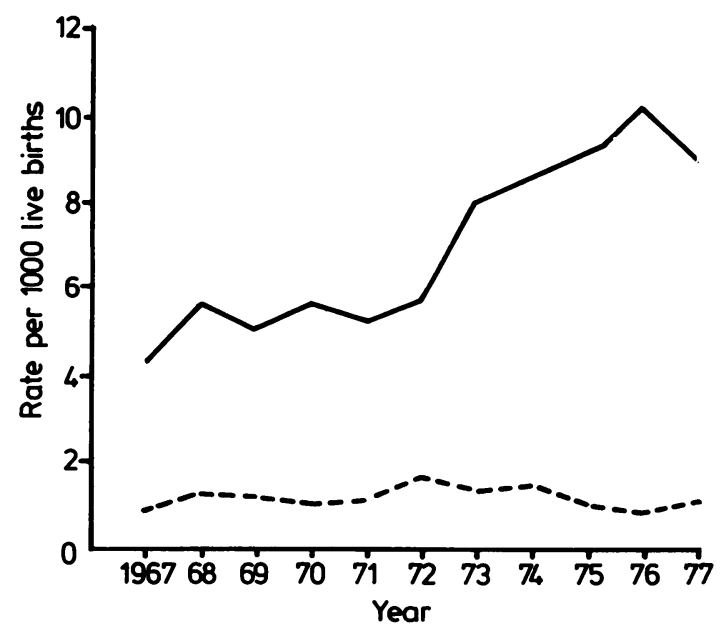

Fig. 1 Birthweight specific prevalence of cerebral palsy for infants with birth weights $\leqslant 2500 \mathrm{~g}(\longrightarrow)$ and $>2500 \mathrm{~g}(---)$. upward trend in prevalence among the low birthweight group $\left(\chi_{\mathrm{IDF}}^{2}\right.$ for trend 11.46, $\left.\mathrm{p}<0.001\right)$, but no trend was observed for infants of normal birth weight $\left(\chi_{\mathrm{IDF}}^{2}\right.$ for trend $0 \cdot 21$, not significant). Higher prevalences were observed if the denominator used was the number of neonatal

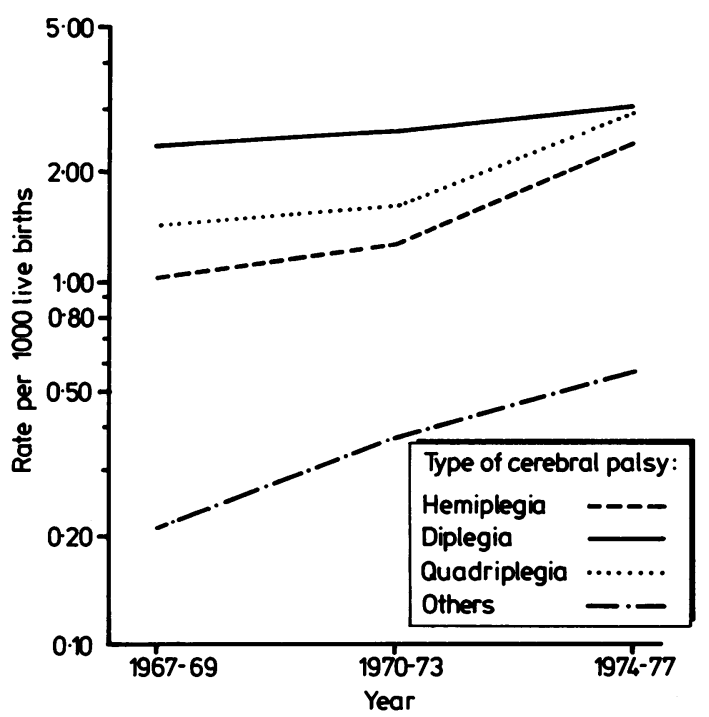

Fig. 2 Trend by type of cerebral palsy for low birthweight $(\leqslant 2500 \mathrm{~g})$ infants.

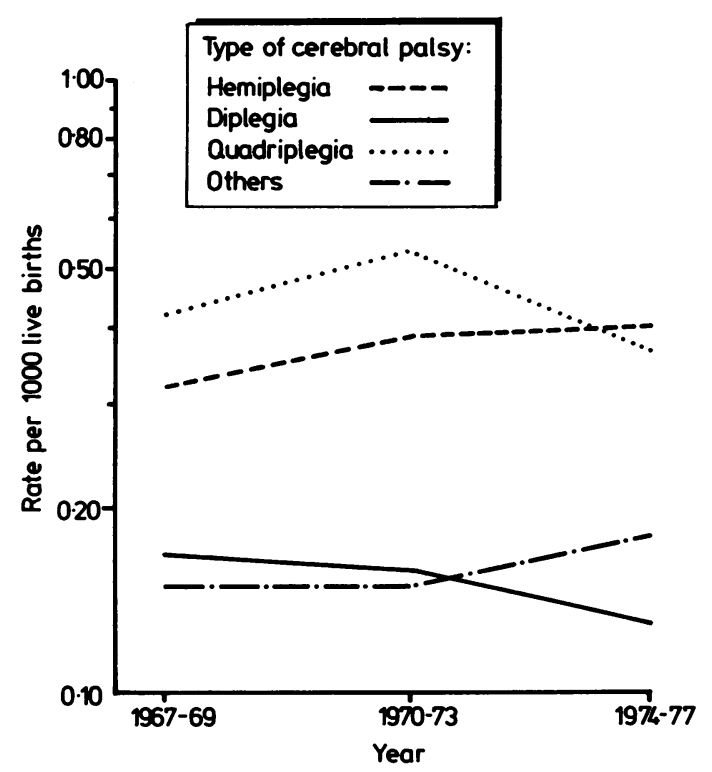

Fig. 3 Trend by type of cerebral palsy for normal birthweight $(<2500 \mathrm{~g})$ infants. 
survivors; the trends in prevalences were similar, however, whether live births or survivors were used for the denominator.

Trends in birthweight specific prevalence according to type of cerebral palsy. The data have been grouped for the years 1967-69, 1970-73, and 197477. Also the mixed spastic syndromes have been combined with the appropriate spastic type-that is, mixed hemiplegia with spastic hemiplegia, mixed diplegia with diplegia, and mixed quadriplegia with quadriplegia. Among low birthweight infants-the upward trend was evident in all types of cerebral palsy (Fig. 2). In none, however, was the trend significant, though this may have been a statistical type II error because the numbers in the samples were small. In contrast, no consistent pattern was obvious in the trend among infants of normal birth weight (Fig. 3).

Thus the overall increase in prevalence among low birthweight infants was the composite result of an increase in prevalence of each type of cerebral palsy.

\section{Discussion}

The recrudescence of interest in the epidemiology of cerebral palsy has occurred primarily because of its possible use as an outcome measure for evaluating neonatal special and intensive care. The use of epidemiology in this manner, however, has its problems.

It is tempting to attribute the recent rise of cerebral palsy among low birthweight infants to the development of neonatal special and intensive care and consider them casualties of technological development ${ }^{7}$ but caution must be exercised before coming to this conclusion. If cerebral palsy is an undesired side effect of special care two plausible explanations could be invoked. The immature infant, though born with an intact brain, may, because of its immaturity, be liable to a variety of insults, such as intraventricular haemorrhage and cerebral ischaemia, with its consequent neurological deficit. Implicit in this explanation is that special/intensive care is a cause of cerebral palsy and directly affects the incidence of the syndrome. The alternative explanation is that the neurological deficit is present before birth as a result of genetic or other factors but that improvements in obstetric and neonatal care ensure that these infants now survive so that the prevalence, though not the incidence, of cerebral palsy is affected.

From the epidemiological standpoint it is crucial to distinguish between these alternatives as the prevention of the disability and ultimately an understanding of the aetiology is dependent on knowledge of the incidence. The observation that the increase in prevalence among low birthweight infants occurred with all types of cerebral palsy suggests that the latter explanation is more plausible - that is, that the incidence has not changed but that improved survival rates, particularly among low birthweight infants, have increased prevalence. Furthermore, it is inherently implausible that neonatal intensive care is aetiologically implicated in all forms of the disease.

A third possible explanation of the upward trend in prevalence of cerebral palsy among low birthweight infants relates to an improvement in the completeness of ascertainment. A deficiency in ascertainment is to be expected when the use of health service facilities determines whether an affected child is registered as a case. Such a failure of ascertainment is particularly liable to occur with those children who are least severely affected. The very successes of neonatal intensive care have generated an increasing interest in the routine follow up of low birthweight infants and ensures that the milder cases of cerebral palsy among this group may now be diagnosed and registered, whereas previously they would have been missed, a phenomenon that has been termed 'diagnostic access' bias. ${ }^{13}$

There is a further reason for not attributing the increase in prevalence of cerebral palsy among low birthweight infants to the development of intensive care. Essentially, the period covered by the survey reported here predates the development of intensive care in the Mersey region; a consultant neonatologist was not appointed until 1980. In support of this, if artificial ventilation is used as an index of intensive care, of the 198 infants with cerebral palsy born weighing $\leqslant 2500 \mathrm{~g}$ during the period 1966-77, only 15 were artificially ventilated. Even if it was assumed that all 15 would not have survived without ventilation, the increase in prevalence of cerebral palsy among low birthweight infants would still have been observed.

If the effectiveness of neonatal intensive care is to be monitored the examination of trends in prevalences of cerebral palsy is not adequate for the purpose, and it is preferable to conduct follow up studies of cohorts of low birthweight infants, ideally among geographically delineated groups, to avoid the bias associated with selectivity of admission to hospital.

The authors gratefully acknowledge the funds provided by the Mersey Regional Health Authority Research Committee, which enabled the study to be carried out. 


\section{References}

1 Bax M. Terminology and classification of cerebral palsy. Dev Med Child Neurol 1964;6:295-7.

2 Pharoah POD, Alberman ED. Mortality of low birthweight infants in England and Wales 1953 to 1979. Arch Dis Child 1981;56:86-9.

${ }^{3}$ Lee K, Paneth N, Gartner LM, Pearlman MA, Gruss L. Neonatal mortality: an analysis of recent improvement in the United States. Am J Public Health 1980;70:15-21.

4 Little WJ. On the influence of abnormal parturition, difficult labours, premature birth and asphyxia neonatorum on the mental and physical condition of the child, especially in relation to deformities. Transactions of the Obstetric Society of London 1862;3:293. Reprinted in Cerebral Palsy Bulletin 1958;1:5-36.

5 Drillien CM. Growth and development in a group of children of very low birthweights. Arch Dis Child 1958;33:10-7.

6 Anonymous. The fate of the baby under $1501 \mathrm{~g}$ at birth [Editorial]. Lancet 1979;i:461-3.

7 Chalmers I, Mutch L. Investment in neonatal intensive care and the handicapped survivor bogey. Lancet 1984;ii:469.

8 Hagberg B, Hagberg G, Olow I. Gains and hazards of intensive neonatal care: an analysis from Swedish cerebral palsy epidemiology. Dev Med Child Neurol 1982;24:13-9.

${ }^{9}$ Hagberg B, Hagberg G, Olow I. The changing panorama of cerebral palsy in Sweden 1954-1970: I analysis of general changes. Acta Paediatr Scand 1975;64:187-92.

${ }^{10}$ Hagberg B, Hagberg G, Olow I. The changing panorama of cerebral palsy in Sweden: IV epidemiological trends 1959-78. Acta Paediatr Scand 1984;73:433-40.

"Stanley FJ. An epidemiological study of cerebral palsy in Western Australia 1956-1975: I changes in total incidence of cerebral palsy and associated factors. Dev Med Child Neurol 1979;21:701-13.

12 Jarvis SN, Holloway JS, Hey EN. Increase in cerebral palsy in normal birthweight babies. Arch Dis Child 1985;60:1113-21.

13 Sackett DL. Bias in analytic research. J Chronic Dis 1979;32: 51-63.

Correspondence to Professor P O D Pharoah, Department of Community Health, University of Liverpool, P O Box 147, Liverpool L69 3BX, England.

Received 24 November 1986 